\title{
Navigating the road to personalized medicine: Can we believe?
}

\author{
Gideon M. Hirschfield MB PhD, Christopher I. Amos PhD, Katherine A. Siminovitch MD
}

Previously published at www.cmaj.ca

"P ersonalized medicine" is a phrase now reverberating across the health care sector, capturing the hope that genomic sciences will provide the capability to individualize and thereby optimize medical care. ${ }^{1}$ This vision builds on the success of genomic sciences - a field that gave us, just 10 years ago, the complete human genome sequence and that continues to produce an extraordinary repertoire of technical and computational tools. Most recently, these technologies have enabled an unprecedented rate of discovery of common disease genes as well as dramatic reductions in resequencing time and costs that have rendered feasible the $\$ 5000$ (and almost certain soon-to-be \$1000) genome. ${ }^{2}$

Without a doubt, genomic technology has delivered - but can it deliver on the promise of personalized health care? Yazdanyar and colleagues have reported on their analysis of the contribution of NOD2 (nucleotide binding oligomerization domain 2)/CARD15 (caspase recruitment domain 15) gene variants to the risk for Crohn disease. ${ }^{3}$ Their findings provide an important reminder of the challenges inherent to translating meaningful medical discovery into meaningful clinical benefit.

Yazdanyar and colleagues analyzed the extent to which carriage of one or two copies of NOD2/CARD15 alleles conferring risk of Crohn disease correlated with the development of the disease in the Danish general population. The association of the NOD2/CARD15 gene with Crohn disease is particularly appropriate for such a study, because it has been widely replicated in independent case-control studies of white patients with the disease. The data suggest that these alleles have a substantial influence on risk (odds ratio [OR] of about 2.4 for heterozygous and 17.0 for homozygous and compound heterozygous carriers, respectively, versus noncarriers of risk alleles). ${ }^{3}$ In addition, the biologic roles of the NOD2/CARD15 protein are consistent with an important role for NOD2/CARD15 signaling in the pathogenesis of Crohn disease..$^{4-6}$

Capitalizing on the availability of a large population with well-curated longitudinal health records collected for over 30 years, Yazdanyar and colleagues genotyped 43596 Danish people for the three major NOD2/CARD15 variants (Arg702Trp, Gly908Arg and Leu1007fsinsC) associated with Crohn disease. They then calculated penetrance of the risk alleles on the basis of the fraction of people in whom the disease developed before 50 years of age. In contrast to results of

\section{Key points}

- The causes of common diseases include many factors, and the effects of any single gene or gene variant on risk are usually small.

- Population-based studies are important in defining more accurately the risk effects of disease alleles identified by case-control association studies.

- Knowledge of the gene variants underpinning common diseases is critical to clarifying the pathogenesis of these conditions, but major challenges remain in translating such information into a personalized health care paradigm.

previously published case-control analyses, Yazdanyar and colleagues found an OR of 1.2 for heterozygous and 3.3 for homozygous and compound heterozygous carriers of risk alleles. Penetrance at age 50 years of the risk genotype for Crohn disease was $0.3 \%$ (95\% confidence interval [CI] 0.29 0.31 ) for heterozygous carriers and $1.5 \%$ (95\% CI 1.40-1.60) for homozygous and compound heterozygous carriers.

Should we be surprised by the apparent discrepancy between the estimates of genotype risk gleaned from a population-based versus case-control association study? Clearly, although the case-control design is valuable in identifying risk alleles for common multigenic disease, it can be inherently biased by the deliberate analysis of people with the disease. As for most common diseases, the genetic contribution to Crohn disease is complex, with the risk for disease potentially engendered by at least 30 gene variants. ${ }^{7}$ The contribution of any one of these variants to risk is relatively small. However, it could be amplified in the context of a patient cohort in whom Crohn disease has already been diagnosed compared with the general population if the former had been enriched for any subphenotype that increases genetic risk or for other risk alleles for the disease that might enable higher penetrance of the risk allele under study.

Gideon Hirschfield is from the Liver Centre, Toronto Western Hospital, Toronto, Ont. and the Department of Medicine, University of Toronto, Toronto, Ont. Christopher Amos is from the Department of Epidemiology, University of Texas, and M.D. Anderson Cancer Center, Houston, USA Katherine Siminovitch is from the Department of Medicine, University of Toronto; Mount Sinai Hospital; Samuel Lunenfeld Research Institute; and Toronto General Research Institute, Toronto, Ont.

CMAJ 2010. DOI:10.1503/cmaj.100300

All editorial matter in CMAJ represents the opinions of the authors and not necessarily those of the Canadian Medical Association. 
If the NOD2/CARD15 variants were associated with severity as well as risk for Crohn disease (as has been suggested in some studies), the case-control study design may also upwardly bias the estimates of genotype risk by including the more severe cases most likely to be followed at tertiary care centres. Similarly, relatives of patients with Crohn disease may be more carefully monitored at such centres in conjunction with family studies, again potentially biasing penetrance estimates in such referral populations. Prospective population studies avoid these issues and are generally preferred in genetic epidemiologic studies. However, this approach also has potential limitations - in particular, the possible failure to identify all patients with a disease (in this case, Crohn disease) in the population, thus yielding falsely low estimates of penetrance.

Although the possibility of underestimated penetrance in Yazdanyar and colleagues' study cannot be formally excluded, the effects of any single gene or gene variant on risk are generally small in common diseases. Moreover, even in so-called monogenic disorders — such as $\beta$-thalassemia, hypertrophic cardiomyopathy and many of the other mendelian-inherited diseases - the association of identical mutations in a single gene with highly diverse phenotypes reinforces the complexity inherent in predicting risk of disease where the cause and, by extension, penetrance of any one risk allele are shaped by multiple genetic and environmental interactions. ${ }^{8-10}$ This study's findings caution us about interpreting estimates of risk from case-control association data sets and reinforce previous contentions that NOD2/ $C A R D 15$ genotyping has no value in identifying people at risk for Crohn disease. Moreover, because the NOD2/CARD15 variants are thought to account for only $20 \%$ of genetic susceptibility to Crohn disease and their penetrance appears to be so low, the predictive value of NOD2/CARD15 genotyping is insufficient to warrant testing first-degree relatives of patients with the disease. This situation could change with a more complete understanding of the etiopathogenesis of Crohn disease that enables reliable risk prediction or with pharmacogenomic data linking NOD2/CARD15 variant genotypes to specific drug responses.
At a more general level, the data from Yazdanyar and colleagues' study reinforce much of the current skepticism about the future of personalized medicine, thereby reminding us that genomic technology has dramatically outpaced understanding of human genetics and that, as aptly noted 10 years ago by Sir David Weatherall, "Relating genotype to phenotype is the challenge for genetic medicine over the next century." "\$,11 As each of us anticipates knowledge of our personal genome, we should appreciate the difficulties inherent to meaningful clinical application of such information while relishing the opportunity to make it so.

Competing interests: None declared.

Acknowledgement: Dr. Siminovitch is a recipient of a Canada Research Chair Award and holds the Sherman Family Research Chair in Genomic Medicine.

\section{REFERENCES}

1. Ginsburg GS, Willard HF. Genomic and personalized medicine: foundations and applications. Transl Res 2009;154:277-87.

2 Service RF. Gene sequencing. The race for the $\$ 1000$ genome. Science 2006;311:1544-6.

3. Yazdanyar S, Kamstrup PR, Tybjaerg-Hansen A, et al. Penetrance of NOD2/CARD15 genetic variants in the general population. CMAJ 2010;182:661-5.

4. Economou M, Trikalinos TA, Loizou KT, et al. Differential effects of NOD2 variants on Crohn's disease risk and phenotype in diverse populations: a meta-analysis. Am J Gastroenterol 2004;99:2393-404.

5. Gasche C, Grundtner P. Genotypes and phenotypes in Crohn's disease: Do they help in clinical management? Gut 2005;54:162-7.

6. Rosenstiel P, Sina C, Franke A, et al. Towards a molecular risk map - recent advances on the etiology of inflammatory bowel disease. Semin Immunol 2009;21:334-45.

7. Barrett JC, Hansoul S, Nicolae DL, et al. Genome-wide association defines more than 30 distinct susceptibility loci for Crohn's disease. Nat Genet 2008;40:955-62.

8. Weatherall DJ. Single gene disorders or complex traits: lessons from the thalassaemias and other monogenic diseases. BMJ 2000;321:1117-20.

9. Arad M, Seidman JG, Seidman CE. Phenotypic diversity in hypertrophic cardiomyopathy. Hum Mol Genet 2002;11:2499-506.

10. Wolf U. Identical mutations and phenotypic variation. Hum Genet 1997;100:305-21.

11. McGuire AL, Cho MK, McGuire SE, et al. Medicine. The future of personal genomics. Science 2007;317:1687.

Correspondence to: Dr. Katherine A. Siminovitch, Mount Sinai Hospital, 600 University Ave., Rm. 778D, Toronto ON M5G 1X5; ksimin@mshri.on.ca 